\title{
Ensuring patients are able to optimise pre- and post-operative oral hygiene
}

More and more people are seeking dental implants as the gold standard solution for partial or complete edentulism. Aside from delivering the highest quality, evidence-based surgical and restorative solutions, it is also the role of the practitioner to ensure that patients appreciate their own responsibility to look after their implants, immediately postoperative and in the long-term. This means making sure that they understand exactly what to do and when for the days prior to and following implant placement. It also involves impressing upon them the importance of regular and meticulous cleaning for as long as they want to keep the implant looking good and functioning as required.

There are several potential barriers to effective patient compliance with pre- and post-operative hygiene instructions that must be prepared for and overcome. The patient's willingness to maintain a sufficient at-home routine is one - even if instructions are only temporary. Their ability to do what is asked of them is another. For the latter, recommending clinically proven but easy to use products that will support patients'

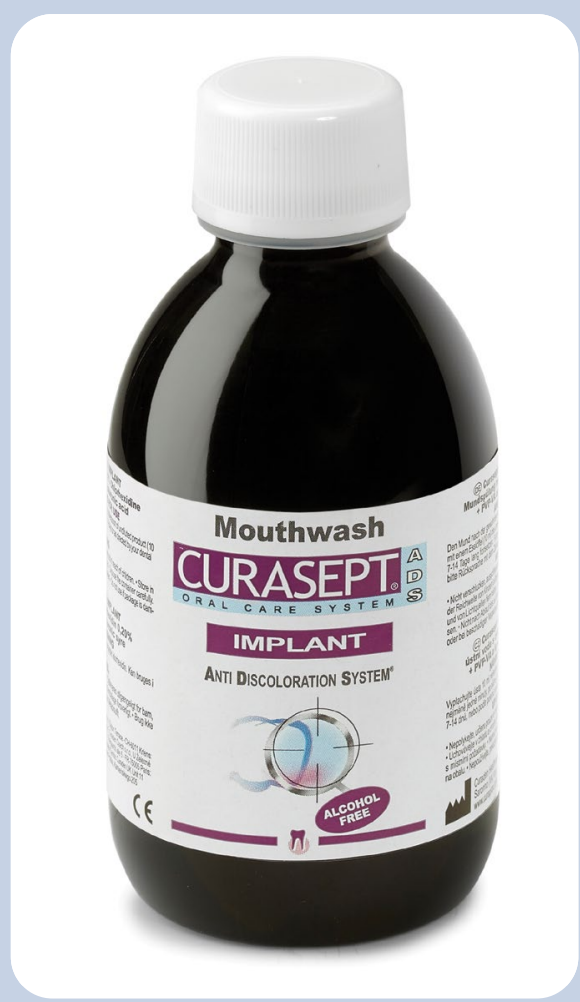

homecare regimes, minimise the risk of infection during surgery and encourage fast and effective post-operative healing is key.

This is where the Curasept ADS Implant mouthwash, available through J\&S Davis in the UK, offers a helping hand. It is specifically formulated to support implant therapy and facilitate the very best outcomes for the patient.

CHX - all the good, none of the bad Like all Curasept ADS products, Curasept ADS Implant contains the patented Anti Discolouration System that minimises the risks of staining or taste disturbance that are often associated with other products containing chlorhexidine (CHX). This means it offers all the plaque-fighting benefits of $\mathrm{CHX}$, without any of the side effects. It's no wonder this is the go-to range of non-staining CHX solutions on the market right now!

\section{A little something extra}

Curasept ADS Implant also contains innovative copolymer PVP-VA and hyaluronic acid to further enhance the positive impact it has for implant patients.

PVP-VA (more commonly known as Poly Vinyl Pyrrolidone Vinyl Acetate) features mucoadhesive properties that facilitate the creation of a protective film over oral structures. This prevents the accumulation of bacteria on the teeth and gums in order to slow its growth and avoid peri-implant infections. The hyaluronic acid helps to further protect the mucous membrane. Its mechanical moisturising and re-epithelising actions accelerate soft tissue healing and tissue repair.

As such, Curasept ADS Implant promotes patient comfort and minimises the risk of infection while also encouraging good soft tissue aesthetics. The antiplaque action is also important in the fight against peri-implant diseases, helping to keep the gingiva healthy and infection-free for uneventful surgery, healing and treatment success. For added benefit, the mouthwash contains xylitol as well, which has been shown to help prevent caries and decay.

\section{Patient convenience}

Perhaps best of all, Curasept ADS Implant is super easy for patients to use. All they have to do is rinse their mouth with a tablespoon of product for at least a minute a day. And that's it. No complicated techniques or uncomfortable and expensive products necessary. Just swirl and spit. Curasept ADS Implant is generally recommended for use one week prior to implant placement and one-week post-surgery for the very best results.

\section{Willing and able}

With good communication and education, patients will be willing to perform extra steps to look after their mouths immediately postimplant placement. To ensure they are also able to meet high oral hygiene standards, it's crucial to give them effective and simpleto-use products as well. The Curasept ADS Implant mouthwash is the ideal solution in many situations, offering added peace of mind for patient and practitioner alike.

If you'd like any further details on this or any of the other products within the Curasept ADS range, J\&S Davis are here to help. Our friendly team can provide any information or support you or your patient need.

Commenting on the service she has received, Gill Cliffe - a dental hygienist from Yorkshire - says: 'The service from J\&S Davis has been fantastic over the years, which is why I keep coming back and will continue to do so. My representative has been exemplary and is definitely a huge asset to the company'.

Dr Makbule Sipahi Ogretme from Locksbottom comments: 'Communication with J\&S Davis has always been easy and now the connections on social media make this even better. Both the representatives I've worked with have been superb. I'd like to thank them for great customer services and being helpful all the time'.

For more information on the industryleading products available from J\&S Davis, visit www.js-davis.co.uk, call 01438747344 or email jsdsales@js-davis.co.uk. 